\title{
RESOLVED STRUCTURE IN THE NUCLEAR REGION OF THE ULTRALUMINOUS INFRARED GALAXY MRK 273
}

\author{
J.H. KNAPEN, S. LAINE, J.A. YATES AND A. ROBINSON \\ Department of Physical Sciences, University of Hertfordshire, \\ Hatfield, Herts AL10 9AB, UK
}

A.M.S. RICHARDS

Nuffield Radio Astronomy Laboratories, University of Manchester, Jodrell Bank, Macclesfield, Cheshire SK11 9DL, UK

AND

R. DOYON AND D. NADEAU

Observatoire du Mont Mégantic and Département de Physique, Université de Montréal, C.P. 6128, Succursale Centre Ville, Montréal (Québec), HЗС зJ7 Canada

As an example of the power of near-infrared (NIR) Adaptive Optics imaging in the detailed study of central regions of galaxies, we present highresolution $K$-band imaging of Mrk 273, in combination with interferometric radio continuum data.

\section{Introduction}

Ultraluminous infrared galaxies (ULIRGs) are among the brightest known galaxies, some with $L_{b o l} \geq 10^{12} L_{\odot}$, comparable with quasars. They are probably powered by AGNs or massive nuclear starbursts, triggered by galactic mergers. Mrk 273, one of the most luminous ULIRGs, was reported to have multiple structure at the scale of $\sim 1$ arcsec in the NIR (Majewski et al., 1993) and at $3.6 \mathrm{~cm}$ (Condon et al., 1991). We report new $K$-band and radio continuum images, all with resolution better than 150 mas.

\section{Observations}

The $K$-band image was obtained in March 1997 with the CFHT using the adaptive optics system PUEO, and the NIR camera MONICA. The 


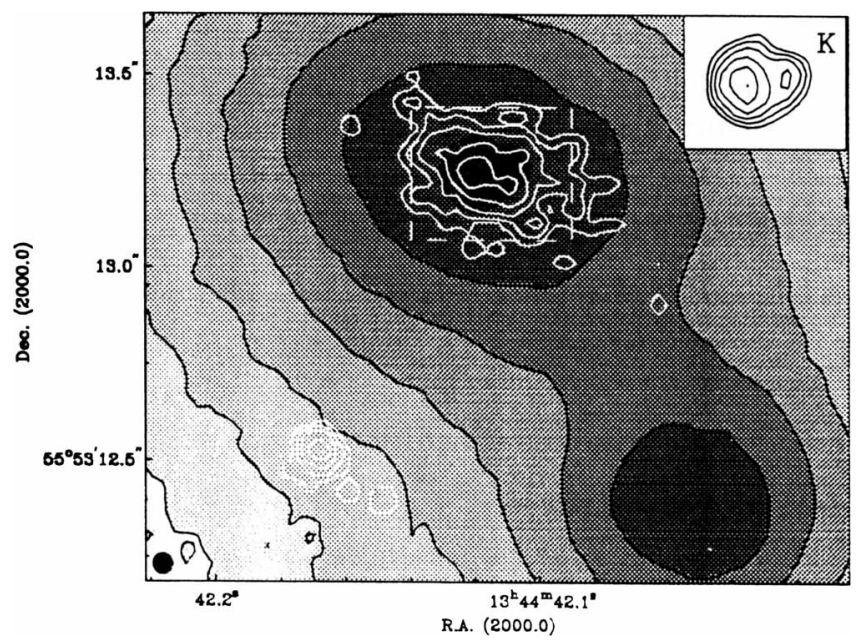

Figure 1. Contours of the $6 \mathrm{~cm}$ radio image overlaid on the $K$ band image of the nuclear region of Mrk 273. The inset shows the twin peaks region in $K$.

corrected spatial resolution is better than 150 mas. Mrk 273 was observed at $6 \mathrm{~cm}$ and $18 \mathrm{~cm}$ with MERLIN, with a beam size of 55 mas at $6 \mathrm{~cm}$ and $170 \mathrm{mas}$ at $18 \mathrm{~cm}$.

\section{Central Morphology of Mrk 273}

The morphology of the central kpc region of Mrk 273 is shown in Fig. 1. The main multiple structure reported before is confirmed, but at 50 mas resolution at $6 \mathrm{~cm}$, the center of the main component is resolved into two peaks, separated by $90 \pm 5$ mas $(\sim 70$ pc). The $K$ image also shows a doublepeaked feature (Fig. 1).

\section{Summary}

We have combined state-of-the-art radio and NIR imaging, all at resolutions around 150 mas, to study the nuclear region of the ULIRG Mrk 273. The radio source within the main component is resolved into a twin-peaked structure. A similar double structure is detected in our $K$-band image. The two peaks could represent distinct core remnants from a recent merger. More discussion can be found in Knapen et al. (1997).

\section{References}

Condon, J.J., Huang, Z.-P., Yin, Q.F., Thuan, T.X. 1991, ApJ 378, 65

Knapen, J. H., Laine, S., Yates, J., Robinson, A., Richards, A.M.S., Doyon, R., Nadeau, D. 1997, ApJL, in press

Majewski, S.R., Hereld, M., Koo, D.C., Illingworth, G.D., Heckman, T.M. 1993, ApJ 402,125 